\title{
DIRECT DETECTION OF LARGE CLOSE-IN PLANETS AROUND THE SOURCE STARS OF CAUSTIC-CROSSING MICROLENSING EVENTS
}

\author{
David S. GrafF and B. Scott Gaudi \\ Departments of Astronomy and Physics, Ohio State University, Columbus, OH 43210; \\ graff.25@osu.edu,gaudi@astronomy.ohio-state.edu \\ Received 2000 April 6; accepted 2000 May 5; published 2000 July 24
}

\begin{abstract}
We propose a direct method to detect close-in Jupiter-sized planets orbiting stars in the Galactic bulge. This method uses caustic-crossing binary microlensing events discovered by survey teams monitoring the bulge to measure light from a planet orbiting the source star. When the planet crosses the caustic, it is more magnified than the source star; its light is magnified by 2 orders of magnitude for Jupiter-sized planets. If the planet has a large radius and is close to the star, it may be bright enough to make a significant deviation in the light curve of the star. Detection of this deviation requires intensive monitoring of the microlensing light curve using a $10 \mathrm{~m}$-class telescope for a few hours after the caustic. This is the only method yet proposed to detect reflected light from close-in planets around stars outside the solar neighborhood.
\end{abstract}

Subject headings: gravitational lensing — planetary systems

\section{INTRODUCTION}

One of the scientific goals of microlensing searches toward the Galactic bulge is the detection of planets orbiting the primary lenses. These searches are conducted in the following manner: one of the microlensing searches, EROS (Glicenstein 2000) or OGLE (Udalski et al. 2000), or the now-terminated MACHO program (Alcock et al. 1996) launches an electronic alert of an ongoing microlensing event. These events are then monitored by follow-up groups such as the PLANET (Albrow et al. 1998), MPS (Rhie et al. 1999), or GMAN (Alcock et al. 1997) collaborations. While the searching teams typically monitor stars about once per day, the follow-up campaigns, with a network of telescopes around the globe, sample much more frequently. A planet orbiting the primary lens with semimajor axis $a$ in the "lensing zone," $0.6-1.5 R_{\mathrm{E}}$ (Mao \& Paczyński 1991; Gould \& Loeb 1992), where the Einstein radius $R_{\mathrm{E}}$ is

$$
R_{\mathrm{E}}=\left(\frac{4 G M}{c^{2}} \frac{D_{L} D_{L S}}{D_{S}}\right)^{1 / 2},
$$

may cause detectable deviations from the standard microlensing light curve (see Sackett 1999 for a review). Here $D_{L}, D_{S}$, and $D_{L S}$ are respectively the distances to the lens, the source star, and between the lens and source stars. For a bulge source lensed by a $0.3 M_{\odot}$ lens, the lensing zone, where one is most sensitive to planets, is in the range 1.2-3.2 AU.

In this Letter, we discuss another means by which a microlensing follow-up experiment may detect a planet, in this case a Jupiter-sized planet close $(a \leq 0.1 \mathrm{AU})$ to the source star. Confounding prior expectations, such close-in large planets are found to be relatively common. They have been detected by several collaborations (Mayor \& Queloz 1995; Cochran et al. 1997; Noyes et al. 1997; see Marcy \& Butler 1998 for a review) and can be found around $\sim 1 \%$ of all stars (Marcy \& Butler 2000).

Since our new method detection is most sensitive to planets close to the source ( $a \lesssim 0.1 \mathrm{AU}$ ), it is complementary to the traditional microlensing light-curve deviation method, which can only detect planets in the lensing zone ( $a \simeq 1-3 \mathrm{AU})$. Unlike other methods of planet detection, such as radial velocity measurements or astrometric shifts, this method can be used to directly ${ }^{1}$ detect planets (by which we mean see reflected light from the planet) in the bulge of our Galaxy, thus allowing us to compare planet formation under conditions different from those in the solar neighborhood.

\subsection{Caustics}

All strong gravitational lenses produce "caustics": configurations of the lens and source in which a true point source would be infinitely magnified. In reality, no source is truly pointlike, and the passage of the source through the caustic allows us to spatially resolve the source. Already, limbdarkening profiles have been measured in sources as far away as the Small Magellanic Cloud (Afonso et al. 2000), and it has been proposed that starspots could be imaged when a source passes through a caustic (Heyrovský \& Sasselov 2000; Han et al. 2000; Bryce \& Hendry 2000). In this Letter, we examine the possibilities of directly detecting light from a planet as it passes through a caustic.

There are several different types of caustics depending on the lens configuration. A single-point lens has a point caustic corresponding to perfect alignment between the source and the lens, when the image of the source is the Einstein ring. These events are rare since they require such perfect alignment. In the case of binary lenses, the caustics form a network of "folds" and "cusps." The caustic structure of binary lenses is cataloged in Schneider \& Weiss (1986) and Erdl \& Schneider (1993). Because caustics form closed curves, a caustic light curve generically has pairs of crossings. The time of the second caustic crossing can often be predicted days in advance, allowing for scheduling of detailed monitoring of the caustic crossing.

The best studied caustic-crossing event, which allows us to illustrate the technology, is MACHO-98-SMC-1 (Alcock et al. 1999), an event that took place in the Small Magellanic Cloud. The MACHO group first sent an alert indicating that a microlensing event was taking place. After the first caustic crossing, the MACHO group launched a second alert with a rough prediction of when the second caustic crossing would occur. As the second caustic crossing approached, predictions of its time

\footnotetext{
${ }^{1}$ Proposed indirect methods to detect bulge planets include the microlensing searches discussed and occultation.
} 
became more accurate so that when it occurred, several groups were able to devote all their telescope time for one night to the observation of this one event. Notably, the passage of the source through the caustic was imaged every $\sim 5$ minutes by the PLANET collaboration (Albrow et al. 1999) from South Africa, followed immediately and with similar sampling frequency by the EROS collaboration (Afonso et al. 1998) from Chile. A combined analysis of this event involving data from the MACHO/GMAN, EROS, PLANET, OGLE, and MPS collaborations was published in Afonso et al. (2000).

Several other caustic crossings have been studied with similar sampling frequency by the MACHO/GMAN and PLANET collaborations (Alcock et al. 2000; M. Albrow et al. 2000, in preparation). Roughly $7 \%$ of all microlensing events are causticcrossing events (Udalski et al. 2000).

Near a fold caustic, the magnification of a single point is (Schneider, Ehlers, \& Falco 1992)

$$
A=A_{0}+\Theta\left(-u_{\perp}\right)\left(u_{\perp} / u_{r}\right)^{1 / 2}
$$

where $u_{\perp}$ is the distance of the source to the caustic normal to the caustic in units of the projected Einstein radius, $u_{r}$ is the length scale of the caustic in units of the projected Einstein radius, $A_{0}$ is the magnification outside the caustic, and $\theta$ is a step function. For typical binary lenses, $u_{r}$ and $A_{0}$ are $O(1)$. The magnification of a uniform disk by a fold caustic is discussed in Schneider \& Weiss (1987). The maximum magnification is $1.4\left(u_{r} / \rho_{*}\right)^{-1 / 2}$, where $\rho_{*}$ is the radius of the stellar disk in units of the projected Einstein radius, while the average magnification during the crossing is $\left(u_{0} / \rho_{*}\right)^{1 / 2}$. Note that since the planet is smaller than the source star, it is more highly magnified.

\section{SCALING RELATIONS}

The fraction of light reflected by a planet can be determined from simple geometry:

$$
f \simeq 10^{-4} p\left(\frac{R_{p}}{R_{\mathrm{Jup}}}\right)^{2}\left(\frac{0.05 \mathrm{AU}}{a}\right)^{2} \phi,
$$

where $p$ is the albedo, $R_{p}$ is the radius of the planet, $a$ is the distance of the planet from the star, and $\phi$ is the fraction of the illuminated surface of the planet visible by the observer, which depends on the inclination and phase of the orbit.

For a source located in the Galactic bulge at $D_{S}=8 \mathrm{kpc}$ and a binary lens at a distance of $D_{L}=6 \mathrm{kpc}$, the typical magnification of a planet of radius $R_{p}$ as it crosses the caustic is

$$
A=10^{2}\left(\frac{R_{\text {Jup }}}{R_{p}}\right)^{1 / 2}\left(\frac{M_{\text {lens }}}{1 M_{\odot}}\right)^{1 / 2}
$$

so that, during the caustic crossing, the fractional deviation of the light curve above that expected when the source star does not have a planet is

$$
\delta_{p} \simeq 1 \% p\left(\frac{R_{p}}{R_{\mathrm{Jup}}}\right)^{1.5}\left(\frac{0.05 \mathrm{AU}}{a}\right)^{2}\left(\frac{M_{\mathrm{lens}}}{1 M_{\odot}}\right)^{1 / 2} \phi
$$

The duration of the planetary caustic crossing will be quite brief. The typical planet diameter-crossing timescale is

$$
t_{p}=12 \frac{R_{p}}{R_{\mathrm{Jup}}} \frac{100 \mathrm{~km} \mathrm{~s}^{-1}}{v_{\perp}} \text { minutes, }
$$

where $v_{\perp}$ is the component of the velocity of the planet normal to the caustic and the line of sight. This velocity is made up of two components: the velocity of the planet around its star and the velocity of the star relative to the caustic. In addition, the planet will not be uniformly illuminated, and thus the width of crossing will depend on the star/planet geometry at the time of the crossing. For example, the caustic crossing from a "quartermoon" planet with the terminator parallel to the caustic will have an effective width that is 2 times smaller than that arising from a quarter-moon planet with the terminator perpendicular to the caustic.

Thus, we see that direct detection of a giant close-in planet requires relative photometry accurate to better than $1 \%$ and a sampling frequency of minutes. In the crowded fields, one must contend with not only the sky background, but also the background due to unresolved stars ("blending"). We use the $I$-band luminosity function of Holtzman et al. (1998) to find the total surface brightness $\mu_{\text {tot }}$ due to unresolved stars assuming a pointsource function (PSF) size of $1.8 \operatorname{arcsec}^{2}$ and the sky background, which we assume to be $\mu_{\text {sky }}=19.5 \mathrm{mag} \mathrm{arcsec}^{-2}$. We find $\mu_{\mathrm{tot}}=19.3 \mathrm{mag} \operatorname{arcsec}^{-2}$. The Poisson noise for $I=$ 19.7 (a G0 V star at $8 \mathrm{kpc}$ with $1 \mathrm{mag}$ of extinction), assuming an overall throughput of 0.1 , a background of $\mu_{\text {tot }}=19.3 \mathrm{mag}$ $\operatorname{arcsec}^{-2}$, and a PSF size of $1.8 \operatorname{arcsec}^{2}$, is

$$
\sigma \simeq 0.7 \%\left(\frac{t_{\exp }}{1 \text { minute }}\right)^{-1 / 2}\left(\frac{D}{10 \mathrm{~m}}\right)^{-1}
$$

where $t_{\exp }$ is the total exposure time and $D$ is the diameter of the telescope. Therefore, for $t_{p}=t_{\exp }=12$ minutes, the planet will be detected with a signal-to-noise ratio of $\delta / \sigma \approx 5$ for a $10 \mathrm{~m}$ telescope.

In addition to the caustic crossing, one may be able detect the planet in the precrossing phase. Ultimately, the magnification of the planet inside the crossing depends on the geometry of the lens, but can reach a factor of typically around 10. In this case, before the planet crosses the caustic, the source will be approximately $0.1 \%$ brighter than after the caustic crossing. This brightening lasts much longer than the caustic crossing, $\Delta t \approx 20 \mathrm{hr}\left(a_{\perp} / 0.05 \mathrm{AU}\right)\left(100 \mathrm{~km} \mathrm{~s}^{-1} / v_{\perp}\right)$, where $a_{\perp}$ is the planet-star distance normal to the caustic. Since of order 50 times more measurements will be taken of this deviation than of the caustic, but the deviation is of order 10 times smaller, the precaustic component will have a signal-to-noise ratio of roughly half that of the planetary caustic crossing.

This level of photometric precision and temporal sampling will challenge present-day microlensing searches, but may be possible with future microlensing follow-up searches.

\section{SAMPLE LIGHT CURVES}

In the previous section, we derived simple scaling relations to estimate the magnitude of the deviation one might expect during a caustic-crossing event from a close-in large-radius planet orbiting the source. We now present a few sample light curves to confirm our estimates from $\S 2$ and illustrate the effect.

We adopt typical lens parameters for events toward the Galactic bulge: we assume the lens is an equal-mass binary at 


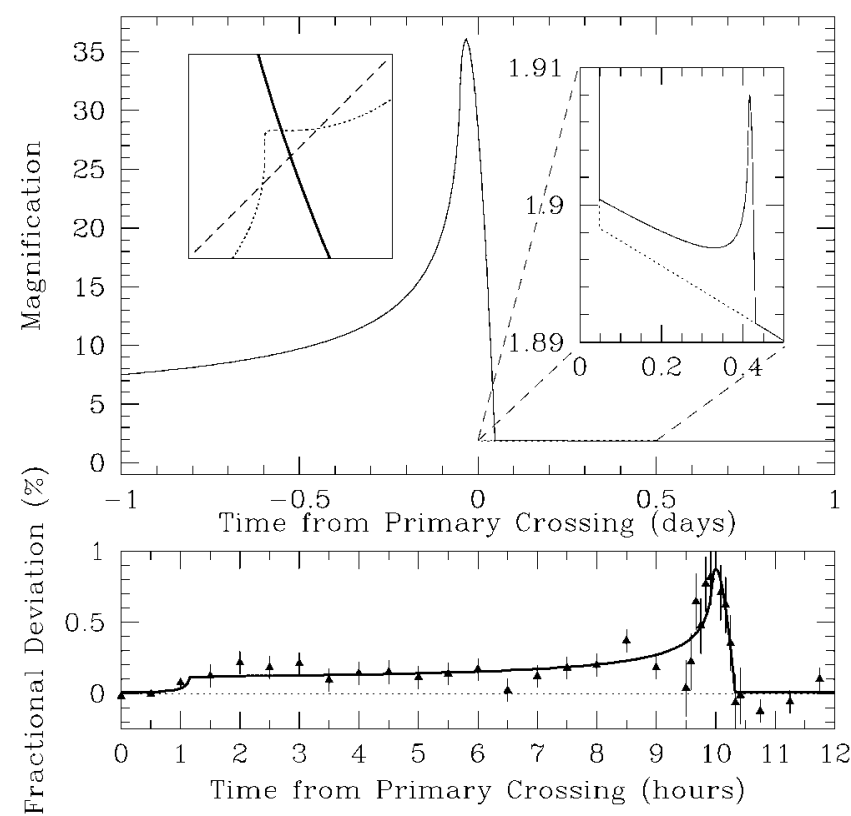

FIG. 1.-Top: Magnification as a function of time from the primary caustic crossing. The lens is an equal-mass binary at $6 \mathrm{kpc}$ with total mass $1 M_{\odot}$. The source system is an analog of HD 209458 at $8 \mathrm{kpc}$ : a G0 V primary with a planet of radius $1.27 R_{\text {Jup }}$ and semimajor axis $a=0.0467 \mathrm{AU}$. The top-left inset shows the geometry of the source system trajectory: the dashed line shows the path of the primary, while the dotted line is the path of the secondary. The solid line is the caustic. The right inset shows a blow-up of the light curve immediately after the primary caustic crossing both with (solid curve) and without (dashed curve) the planet. Bottom: The fractional deviation from the single-source (i.e., no planet) light curve as a function of time from the primary caustic crossing. The points with error bars are simulated photometric measurements for a $I \simeq 20 \mathrm{mag}$ star assuming 5 minute exposures on a $10 \mathrm{~m}$ telescope and photon noise-limited precision. Data outside the planet caustic crossing has been binned in 30 minute intervals. In this case, the deviation would be detected with a signal-to-noise ratio of 20 .

$6 \mathrm{kpc}$ with total mass $1 M_{\odot}$, projected separation of $1 R_{\mathrm{E}}$, and transverse velocity $v=150 \mathrm{~km} \mathrm{~s}^{-1}$. We begin by assuming the source system is an analog of HD 209458 at $8 \mathrm{kpc}$ : a G0 V primary with a planet of radius $1.27 R_{\text {Jup }}$ and semimajor axis $a=0.0467 \mathrm{AU}$ (Charbonneau et al. 2000) and the planet orbit plane is face-on to the observer. The resulting light curve is shown in Figure 1. Confirming our estimates from $\S 2$, we find that the fractional deviation is $\sim 0.1 \%$ for the first $\sim 9 \mathrm{hr}$ after the primary caustic crossing and $0.5 \%-1.0 \%$ in the $\sim 30$ minute interval during which the planet is crossing the caustic.

To illustrate the diversity of features possible, in Figure 2 we show light curves one would expect under various assumptions about the lens and source systems. If the planet is very close to the primary $a \leqq 0.03 \mathrm{AU}$, relatively large $R_{p} \gtrsim 1.5 R_{\text {Jup }}$, or the lens has a low mass $M_{\text {lens }} \leqslant 0.3 M_{\odot}$, then the magnitude of the deviation can be quite substantial $\left(\delta_{p} \geq 1 \%\right)$ and may be detectable by current microlensing follow-up surveys (at least for bright stars). Also, if the period of the planetary orbit is smaller than the time it takes for diameter of the orbit to traverse the caustic, then the planet will cross the caustic three (or more) times. As we discuss in $\S 4$, this would allow one to extract additional information about the planet.

\section{DISCUSSION}

The current microlensing follow-ups are only marginally able to detect planets around bright source stars by this method.
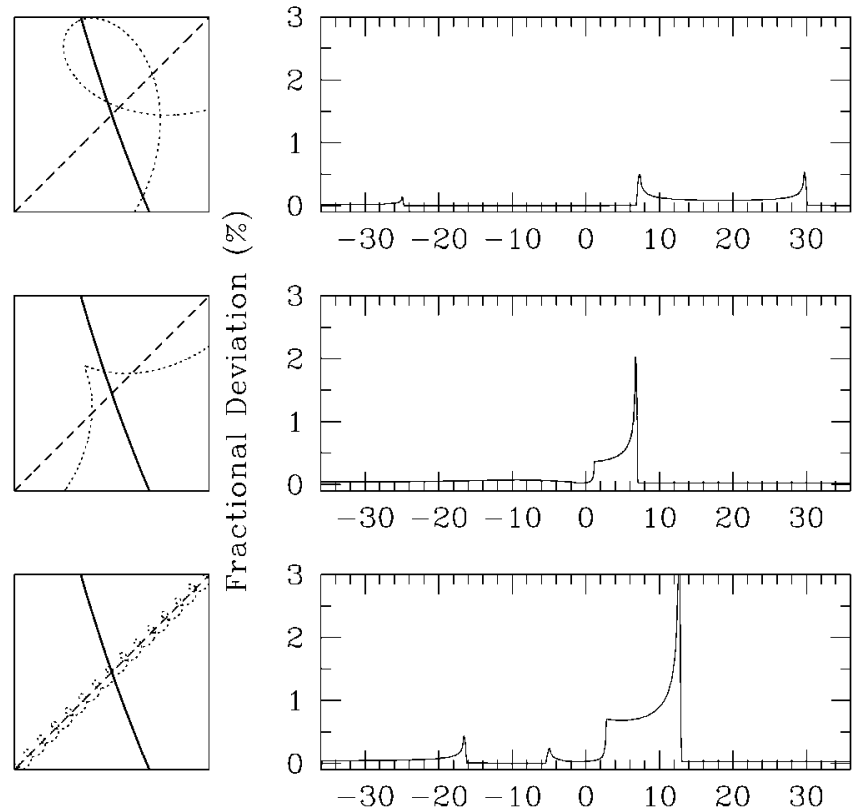

Time from Primary Crossing (hours)

FIG. 2.-Right panels: Fractional deviation from the single-source (i.e., no planet) light curve as a function of time from the primary caustic crossing for three different assumptions of the lens and/or planet parameters. In all cases, the primary is a G0 V star at $8 \mathrm{kpc}$. For each panel, the geometry of the source system trajectory is shown to the left. Line types are the same as in Fig. 1. Top panels: Lens mass $M_{\text {lens }}=0.3 M_{\odot}$, lens distance $D_{L}=7 \mathrm{kpc}$, and lens transverse velocity $v=60 \mathrm{~km} \mathrm{~s}^{-1}$. The planet has a radius $R_{p}=1.27 R_{\text {Jup }}$ with semimajor axis $a=0.0467 \mathrm{AU}$ (as in Fig. 1). Middle panels: $M_{\text {lens }}=$ $0.3 M_{\odot}, D_{L}=6 \mathrm{kpc}, v=150 \mathrm{~km} \mathrm{~s}^{-1}, R_{p}=1.5 R_{\text {Jup }}$, and $a=0.03 \mathrm{AU}$. Bottom panels: $M_{\text {lens }}=1.0 M_{\odot}, D_{L}=2 \mathrm{kpc}, v=20 \mathrm{~km} \mathrm{~s}^{-1}, R_{p}=1.5 R_{\text {Jup }}$, and $a=0.03 \mathrm{AU}$.

They will only detect the closest in, largest, slowest moving planets around relatively bright sources, with low signal-tonoise ratios and only a handful of photometric measurements. However, these follow-up experiments currently use only $1 \mathrm{~m}-$ class ground-based telescopes and are further hindered by their relatively poor seeing in extremely crowded fields. A followup program involving one night on a $10 \mathrm{~m}$-class telescope with adaptive optics should be able to provide millimagnitude photometry of 19th magnitude bulge sources with 5 minute time resolution and have a good chance of finding a close in Jupitersized planet around the source if one is there to be found.

How many planets per year could be detected? Currently, $\sim 50$ events are alerted toward the Galactic bulge each year; future upgrades may provide as many as $\sim 500$ alerts per year. Caustic-crossing binaries comprise $\sim 7 \%$ of all events (Udalski et al. 2000). Assuming that the fraction of stars in the bulge with close planets is similar to the local neighborhood, $\sim 1 \%$, than we would expect to detect of order 1 planet per year. This would require a total $\sim 400 \mathrm{hr}$ per season on $10 \mathrm{~m}$-class telescopes.

Often the event will not be suitable for study: the caustic crossing may occur during full moon or the source star may be highly blended, only contributing a small fraction to the total light. These cases can all be detected days before the second caustic crossing, and a follow-up program can be cancelled before any $10 \mathrm{~m}$ telescope time is wasted on it.

Although the simple detection of a planet around a bulge star is interesting, ultimately one would like to measure physical parameters of the planet: its radius, semimajor axis, and albedo. 
Unfortunately, the observables are complicated functions of not only these parameters, but also the inclination and phase of the orbit and the mass and distance to the lensing system. For example, the timescale of the caustic crossing depends on not only the radius of the planet, but also on the projected velocity of the planet around its star and the phase of the planet (a crescent planet will appear to have a smaller radius than a full planet).

Given excellent data on one caustic crossing, it would be possible to determine the effect of the phase of the planet on the width of the caustic crossing from the shape of the crossing alone. The required level of data will probably not be possible with a $10 \mathrm{~m}$-class telescope, but should be possible with a $100 \mathrm{~m}$ telescope such as the OWL (Gilmozzi et al. 1998). Even then, it still will not be possible to recover the velocity of the planet, and therefore it will be impossible to measure its radius. However, if the transverse velocity of the star normal to the caustic is smaller than the velocity of the planet, it is possible that there will be three caustic crossings. If all three are detected, each with its own time and its own timescale, then the orbit can be solved, and the radius determined, even without inferring the planetary phase from small fluctuations in the light curve.

Irrespective of whether or not the orbit can be solved, planetary caustic crossings act like a telescope with tremendous angular resolution perpendicular to the caustic. The angular dependence of the planetary albedo may be observable. In the future, enormous telescopes such as the OWL may be able to achieve enough temporal resolution to see the bands and red spot on a Jupiter around a star clear across the Galaxy.

\section{CONCLUSIONS}

We have shown that the presence of an HD 209458-like Jupiter-sized close-in planet around the source star of a causticcrossing microlensing event could generate an order $1 \%$ deviation in the light curve. This deviation would be detectable with a $10 \mathrm{~m}$-class telescope making measurements every few minutes over the course of a night. In some cases, the planet will transit the caustic more than once, allowing for complete solution of the orbit and a determination of the radius and albedo of the planet. Follow-up with 100 m-class telescopes may allow for resolution of limb darkening and spots or bands on the surface of these planets.

This Letter profited from useful discussions with Pierre Vermaak, Andrew Gould, and Penny Sackett. This work was supported in part by grant AST 97-27520 from the NSF. B. S. G. acknowledges the support of a Presidential Fellowship from Ohio State University.

\section{REFERENCES}

Afonso, C., et al. 1998, A\&A, 337, L17 2000, ApJ, 532, 340

Albrow, M., et al. 1998, ApJ, 509, 687 1999, ApJ, 512, 672

Alcock, C., et al. 1996, ApJ, 463, L67 1997, ApJ, 491, 436 1999, ApJ, 518, 44 2000, ApJ, submitted (astro-ph/9907369)

Bryce, H., \& Hendry, M. 2000, in Microlensing 2000: A New Era of Microlensing Astrophysics, ed. P. Sackett \& J. Menzies (San Francisco: ASP), in press

Charbonneau, D., et al. 2000, ApJ, 529, L45

Cochran, W. D., et al. 1997, ApJ, 483, 457

Erdl, H., \& Schneider, P. 1993, A\&A, 268, 453

Gilmozzi, R., et al. 1998, Proc. SPIE, 3352, 778

Glicenstein, J.-F. 2000, in Microlensing 2000: A New Era of Microlensing Astrophysics, ed. P. Sackett \& J. Menzies (San Francisco: ASP), in press Gould, A., \& Loeb, A. 1992, ApJ, 396, 104
Han, C., et al. 2000, MNRAS, in press

Heyrovský, D., \& Sasselov, D. 2000, ApJ, 529, 69

Holtzman, J. A., et al. 1998, AJ, 115, 1946

Mao, S., \& Paczyński, B. 1991, ApJ, 374, L37

Marcy, G., \& Butler, R. 1998, ARA\&A, 36, 57

2000, PASP, 112, 137

Mayor, M., \& Queloz, D. 1995, Nature, 378, 355

Noyes, R. W., et al. 1997, ApJ, 483, L111

Rhie, S. H., et al. 1999, ApJ, 522, 1037

Sackett, P. D. 1999, in Planets outside the Solar System: Theory and Observations, ed. J.-M. Mariotti \& D. Alloin (NATO-ASI Ser. C; Dordrecht: Kluwer), 189

Schneider, P., Ehlers, J., \& Falco, E. E. 1992, Gravitational Lenses (Berlin: Springer)

Schneider, P., \& Weiss, A. 1986, A\&A, 164, 237 1987, A\&A, 171, 49

Udalski, A., et al. 2000, Acta Astron., 50, 1 